\title{
Osteoblastic differentiation and stress response of human mesenchymal stem cells exposed to alternating current electric fields
}

\author{
Marie Hronik-Tupaj, William L Rice, Mark Cronin-Golomb, David L Kaplan, Irene Georgakoudi
}

\author{
* Correspondence: marie. \\ tupaj@tufts.edu \\ Department of Biomedical \\ Engineering, 4 Colby Street, \\ Science \& Technology Center, Tufts \\ University, Medford, MA 02155 USA
}

\begin{abstract}
Background: Electric fields are integral to many biological events, from maintaining cellular homeostasis to embryonic development to healing. The application of electric fields offers substantial therapeutic potential, while optimal dosing regimens and the underlying mechanisms responsible for the positive clinical impact are poorly understood.

Methods: The purpose of this study was to track the differentiation profile and stress response of human bone marrow derived mesenchymal stem cells (hMSCs) undergoing osteogenic differentiation during exposure to a $20 \mathrm{mV} / \mathrm{cm}, 60 \mathrm{kHz}$ electric field. Morphological and biochemical changes were imaged using endogenous two-photon excited fluorescence (TPEF) and quantitatively assessed through eccentricity calculations and extraction of the redox ratio from NADH, FAD and lipofuscin contributions. Real time reverse transcriptase-polymerase chain reactions (RT-PCR) were used to track osteogenic differentiation markers, namely alkaline phosphatase (ALP) and collagen type 1 (col1), and stress response markers, such as heat shock protein 27 (hsp27) and heat shock protein 70 (hsp70). Comparisons of collagen deposition between the stimulated hMSCs and controls were examined through second harmonic generation (SHG) imaging.
\end{abstract}

Results: Quantitative differences in cell morphology, as described through an eccentricity ratio, were found on days 2 and days $5(p<0.05)$ in samples exposed to the electric field. A delayed but two fold increase in ALP and col1 transcript was detected by week $2(p<0.05)$ in differentiating hMSCs exposed to an electric field in comparison to the nonstimulated controls. Upregulation in stress marker, hsp27, and type 1 collagen deposition were correlated with this response. Increases in $\mathrm{NADH}$, $F A D$, and lipofuscin were traced in the stimulation group during the first week of field exposure with differences statistically significant on day $10(p<0.05)$. Changes in hsp27 expression correlate well with changes in lipofuscin detected in the stimulation group, suggesting a connection with oxidative stress. Both differentiation factors and electrical stimulation improved hMSC differentiation potential to bone based on calcium deposition on day 28.

Conclusions: Electrical stimulation is a useful tool to improve hMSC osteogenic differentiation, while heat shock proteins may reveal underlying mechanisms, and optical non-invasive imaging may be used to monitor the induced morphological and biochemical changes. 


\section{Background}

In the United States, fractures account for 5.6 million annual musculoskeletal conditions [1]. After one year post-injury, 5-10\% of bone fractures show impaired healing and require additional orthopedic intervention [1]. The clinical practice of orthopedics has empirically used many techniques to establish an optimal environment for skeletal repair [2,3], including grafting, casting, and splinting methods [2]. For more severe fractures, treatments include load-bearing techniques that range from mechanical stress to ultrasound $[4,5]$. Musculoskeletal tissues, including bone and cartilage, respond to biophysical inputs such as electric and electromagnetic fields [6]. Biophysical stimulation is advantageous over pharmacological or chemical therapeutics due to the absence of local toxicity in the surrounding tissue or adverse systemic reactions. To apply biophysical stimulation for clinical use, non-invasive and implantable electromagnetic devices are used, such as bone growth stimulators [7,8]. Non-invasive devices may allow for patient comfort, while implantable devices ensure patient compliance $[9,10]$. Clinical devices employ pulsed electromagnetic fields (PEMF), or direct currents (DC) for healing nonunions and spinal fusions [11,12]. For example, in one clinical study, $76 \%$ of recalcitrant non-unions were healed during treatment employing capacitively coupled (CC) alternating current electric fields [13].

As electromagnetic stimulators are clinically successful for healing bone fractures, we aimed to further examine the impact and mechanisms of electric fields on bone related outcomes through utilizing a human stem cell source. hMSCs have proved to be a competent cell source for tissue engineering applications including bone, cartilage, and adipose tissue regeneration. Furthermore, mesenchymal stem cells have demonstrated a positive response to physical forces [14]. For example, ossicles that are stimulated solely in the mesenchymal stage yield calcium content commensurate with ossicles that are stimulated throughout development [15]. Thus these cells may be more sensitive to electromagnetic field stimulation during bone formation [15].

In the present study, we hypothesized that a $20 \mathrm{mV} / \mathrm{cm} 60 \mathrm{kHz}$ electric field applied for 40 minutes daily would increase biomarker expression and stress response on hMSCs, and would decrease the time to osteogenic differentiation. The rationale for choosing AC electric current at $60 \mathrm{kHz}, 20 \mathrm{mV}$ was based on a series of previous studies that reported increases in osteogenic and chondrogentic differentiation markers, such as TGF- $\beta_{1}$, type 2 collagen, proteoglycan, bone morphogenetic proteins, as well as cell proliferation at this strength [16-20]. A $60 \mathrm{kHz}, 20 \mathrm{mV} / \mathrm{cm}$ electric field strength was used for all studies. Initial electric field effects on differentiating mesenchymal stem cells were observed by examining cell morphological and biochemical changes using nondestructive two-photon excited fluorescence (TPEF) imaging. Metabolic profiles of the cells were tracked to provide insight into whether an increased charge in the surrounding environment alters metabolic pathways, which could in turn impact hMSC osteogenic differentiation and bone healing. Our electric field setup was modeled as two electrodes in parallel separated by a $1 \mathrm{~cm}$ distance. Electrodes were in direct ohmic contact with cell culture media. During stimulation, ionic current was transferred through the culture media across the chamber. To provide insight into the rate and extent of osteogenic differentiation in a low frequency AC electric field, early and mid stage differentiation markers, alkaline phosphatase (ALP) and collagen type I (col1) were assessed at the transcript level. We examined stress responses with heat shock protein 27 (hsp27) 
and heat shock protein 70 (hsp70), due to the ability of these markers to impact osteogenic differentiation and cell metabolism [21-23]. Indeed, heat shock protein responses increased in response to stimulation and were correlated with bone differentiation markers. Finally, for the purpose of examining post-translational col type 1 expression and complete bone differentiation, type 1 collagen and calcium deposition were assessed through second harmonic generation imaging and Alizarin Red staining, respectively.

\section{Methods}

\section{Human mesenchymal stem cell culture}

hMSCs (Tulane University, New Orleans, LA) were from male donors $<25$ years of age and plated for expansion at 5,000 cells $/ \mathrm{cm}^{2}$ in T175 flasks with Dulbecco's Modified Eagle Medium (DMEM) containing $1 \mathrm{~g} / \mathrm{L} \mathrm{D}$-glucose, and $110 \mathrm{mg} / \mathrm{L}$ sodium pyruvate (Invitrogen Corp, Grand Island, NY). DMEM was supplemented with $584 \mathrm{mg} / \mathrm{L} \mathrm{L}$-glutamine, 3,500 mg/L D-glucose, $10 \%$ fetal bovine serum, $1 \%$ penicillinstreptomycin, $0.25 \mathrm{mg} / \mathrm{mL}$ fungizone, $0.1 \mathrm{mM}$ nonessential amino acids, and $1 \mathrm{ng} / \mathrm{mL}$ basic fibroblast growth factor (bFGF) containing $10 \mathrm{mM}$ Tris at $\mathrm{pH} 7.6$ and $0.1 \%$ bovine serum albumin (BSA) (Invitrogen Corp, Grand Island, NY). Cell culture medium was changed two times per week. All experiments used hMSC passages between P2-P6.

\section{Osteogenic differentiation}

Osteogenic differentiation medium included standard hMSC culture medium as listed above with the exception of $1 \mathrm{ng} / \mathrm{mL}$ bFGF. In addition, $0.05 \mathrm{mM} \mathrm{L}$-ascorbic acid 2-phosphate (Sigma-Aldrich, St. Louis, MO), 1,000 nM dexamethasone-water soluble (Sigma-Aldrich, St. Louis, MO), $10 \mathrm{mM}$ glycerol 2-phosphate disodium salt hydrate (Sigma-Aldrich, St. Louis, MO), and $100 \mathrm{ng} / \mathrm{mL}$ bone morphogenetic protein (BMP)-2 (gift from Wyeth, Madison, NJ) was added. Differentiation medium was changed two times per week.

\section{Electrical stimulation chamber design}

The electrical stimulation chamber was modified from previous designs to interface with imaging equipment [24]. Briefly, two $5 \mathrm{~mm}$ long carbon rods (Ladd Research, Williston, VT), $3 \mathrm{~mm}$ in diameter were separated using two $25 \times 7 \times 7 \mathrm{~mm}$ polydimethylsiloxane (PDMS) blocks. PDMS blocks were custom made using a Sylgard 184 Silicone Elastomer Kit (Ellsworth Adhesives, Germantown, WI) in a 10:1 (w/w) ratio of base to curing agent. Two $2 \mathrm{~mm}$ diameter holes having a distance of $10 \mathrm{~mm}$ apart were punched into the PDMS block. A carbon rod was pushed through each hole. A second PDMS block was placed on the opposite ends of the carbon rods for support. Two $8 \mathrm{~cm}$ long platinum wires with 99.995\% purity (Surepure Chemetals, Florham Park, NJ) were secured between each carbon rod and PDMS support to create a strong connection with the rod. Following assembly, PDMS supports, carbon rods, and platinum wire were autoclaved then placed in a $50 \mathrm{~mm}$ diameter poly-d-lysine coated glass bottom Falcon Dish that contained a $14 \mathrm{~mm}$ diameter glass coverslip with a thickness of 0.16-0.18 mm (MatTek Corporation, Ashland, MA) (Figure 1a). For a voltage source, a TENMA Universal Test Center 72 -1005 Function Generator (TENMA Test Equipment, Springboro, $\mathrm{OH}$ ) was connected to the stimulation chamber's platinum wires via 


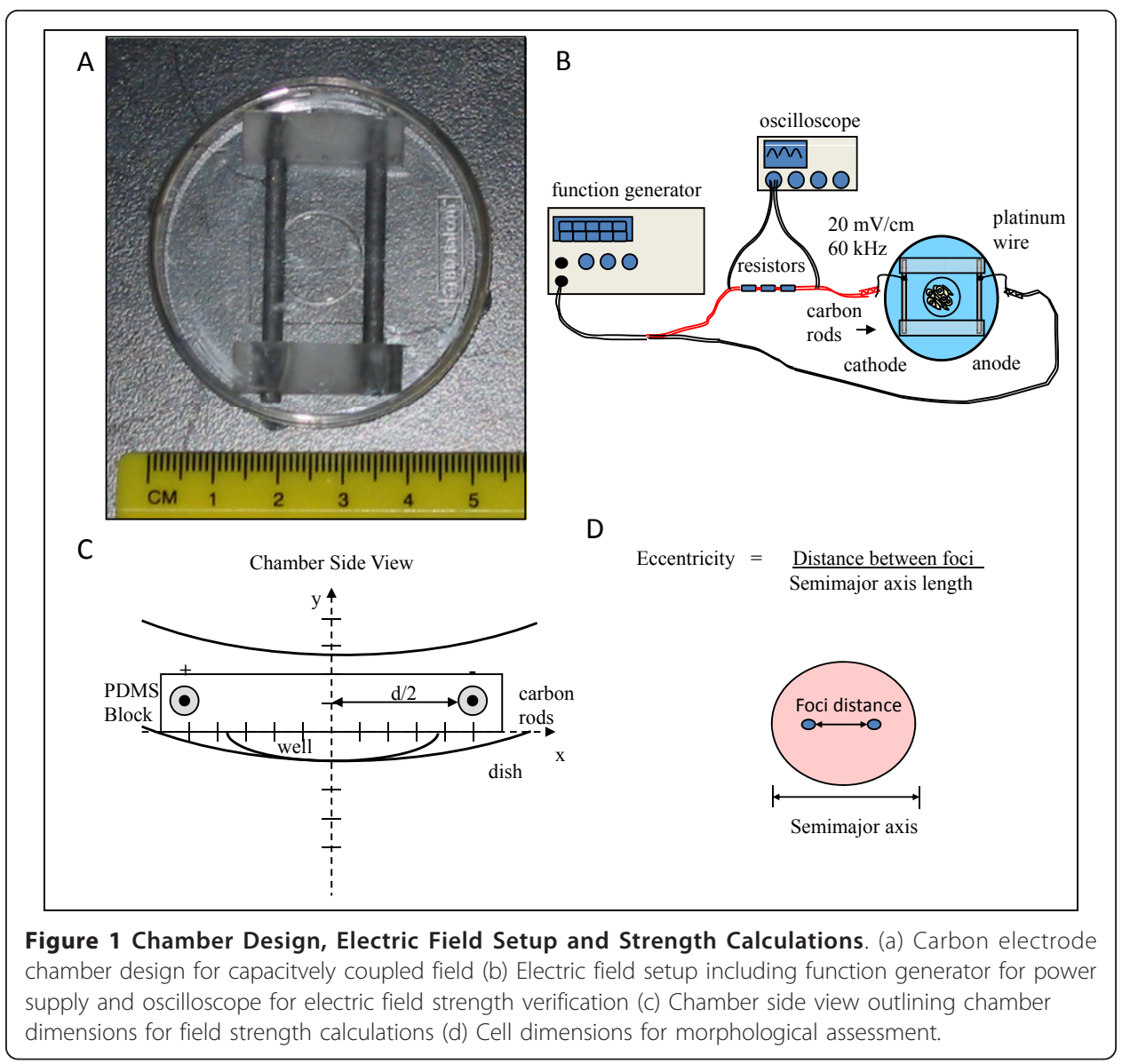

alligator clips and 18 gauge copper wires (resistivity $\rho=1.724 \times 10^{-8} \mathrm{ohm} \mathrm{m}$ ). Three $220 \Omega$ resistors were placed on one of the copper wire leads in series (Figure 1b).

\section{Electric field strength calculations}

Assuming both carbon rods in the chamber are of equal diameter and have a uniform surface charge density, derivations were made using a form of Gauss's Law that calculates electric field strength at any distance, $r$, from an infinite line charge (equation 1).

$$
E=\frac{1}{2 \pi \varepsilon}\left(\frac{\rho}{r}\right)
$$

To adapt Gauss's Law for our own chamber model, we first converted equation 1 from cylindrical coordinates to Cartesian Coordinates and offset each rod by a distance of $\pm \frac{d}{2}$. Electric field intensity at any point $(x, y)$ in the stimulation chamber is the summation of individual charge contributions from both the positively and negatively charged rods (equation 2).

$$
E_{x}=\frac{V_{a b}}{2 \ln \left(\frac{a}{d-a}\right)}\left[\frac{x-\frac{d}{2}}{\left(x-\frac{d}{2}\right)^{2}+y^{2}}-\frac{x+\frac{d}{2}}{\left(x+\frac{d}{2}\right)^{2}+y^{2}}\right]
$$


$\mathrm{V}_{\mathrm{ab}}$ is the voltage potential measured across the carbon rods, $\mathrm{a}$ is the carbon rod radius, $\mathrm{d}$ is the distance between the carbon rods, $\mathrm{x}$ is the distance in the $\mathrm{x}$ direction of the cells from the center of the carbon rods, and $y$ is the distance of cells from the charged rods in the y direction (Figure 1c). For a complete derivation see Appendix. To ensure that $20 \mathrm{mV} / \mathrm{cm}$ electric field strength $\left(E_{\mathrm{x}}\right)$ reached the cells at any point in the glass well, equation 2 was used to identify the voltage potential $\left(\mathrm{V}_{\mathrm{ab}}\right)$ needed at the carbon rods. A $60 \mathrm{MHz} 2213 \mathrm{~A}$ Tektonix oscilloscope (Beaverton, $\mathrm{OH}$ ) was used to measure the voltage across the carbon electrodes in culture media and ensure that a $20 \mathrm{mV} / \mathrm{cm}$ potential strength reached the cells inside the chamber.

\section{Experimental design}

The experimental setup included two study groups; a control group consisting of hMSCs in osteogenic differentiation medium and a stimulation group consisting of hMSCs with differentiation medium and the applied electrical stimulation. All differences in osteogenic differentiating hMSCs exposed to the applied electric field were compared to the control group consisting of osteogenic differentiating hMSCs only. Early gene expression markers and calcium content of a positive hMSC osteoblast control group and negative hMSC adipogenic control groups have been reported previously in our lab [25]. Stem cells were initially seeded at a density 50,000 cells $/ \mathrm{cm}^{2}$ in $150 \mathrm{uL}$ of culture media on individual glass bottom dishes or individual glass bottom dishes with stimulation chambers. Cells were allowed 45 minutes to attach to the bottom of the dishes and then $2-4 \mathrm{~mL}$ of culture or differentiation medium were added per sample to the glass well. All samples were kept at $37^{\circ} \mathrm{C}$ and $5 \% \mathrm{CO}_{2}$. Each day, hMSC samples in the stimulation chambers were exposed to field strengths $20 \mathrm{mV} / \mathrm{cm}$ oscillating at $60 \mathrm{kHz}$. The $60 \mathrm{kHz}$ frequency was chosen as previous studies utilizing alternating current fields reported increases in osteogenic and chondrogenic differentiation markers, such as TGF- $\beta_{1}$, type 2 collagen, proteoglycan, as well as cell proliferation [17-19]. The applied signal was a symmetrical sine wave applied 40 minutes daily for 28 days. Prior to daily stimulation the voltage potential and frequency were measured over the $220 \Omega$ resistors using an oscilloscope to ensure that the applied field strength was the same each day and the same over each sample. A $100 \mu \mathrm{A}$ current was calculated through the $220 \Omega$ resistors according to Ohm's Law. Autoclave paper was placed under each chamber before stimulation to prevent any additional unwanted electrical connections. All electric field stimulations were applied in a laminar flow hood. For consistency, the control samples were placed at room temperature during the time of stimulation. On days 5, 10, 15, and 20 stimulation and control dishes were sacrificed for real time- polymerase chain reaction (RT-PCR). Cellular activity measurements were taken using AlamarBlue on days 5, 10, 15, 20. Two-photon excited fluorescence (TPEF) and second harmonic generation (SHG) images were taken at day 2 and on every fifth day to assess morphological changes, collagen deposition, and metabolic activity. On day 28, Alizarin Red stain was applied to assess calcium content In previous studies, mineralization has been reported within 3-4 weeks of hMSCs undergoing osteogenic differentiation with the addition of (BMP)- 2 to the differentiation media $[26,27]$. The experiment ran for 28 days as this was sufficient time to observe hMSC osteogenic differentiation with the addition of osteogenic differentiation medium and (BMP)-2. 


\section{Morphology}

Two-photon excited fluorescence (TPEF) images based on endogenous fluorescence emission were acquired using a two-photon ready Leica DM IRE2 confocal microscope (Leica, Wetzlar, Germany) and equipped with a Mai Tai solid state tunable (710$920 \mathrm{~nm}$ ) Ti:sapphire laser (Spectra Physics, Mountain View, CA), emitting 100 fs pulses at a rate of $80 \mathrm{MHz}$. TPEF images were taken at 755, 800, and $860 \mathrm{~nm}$ excitation and $455 \mathrm{~nm} \pm 35 \mathrm{~nm}$ and $525 \mathrm{~nm} \pm 25 \mathrm{~nm}$ emission using two non-descanned detectors. Images were acquired with a water immersion, $63 \times$, numerical aperture of 1.2 objective. Incident laser power was approximately $12.5 \mathrm{~mW}$ at $755 \mathrm{~nm}$ and 5.2 $\mathrm{mW}$ at $860 \mathrm{~nm}$. Three areas per sample were imaged from two samples per group. Cell shape and morphological changes were quantitatively described through the eccentricity ratio, defined as the distance between the foci of an ellipse over the semimajor axis length (Figure 1d). As the eccentricity ratio approaches zero, the cell shape becomes rounder; while the ratio is closer to 1 as the cells become more elongated. Eccentricity calculations were done using a program that was created in MATLAB (MathWorks, Natick, MA). Morphological changes were graphed as average eccentricity value vs. time. Thirty cells were assessed at each time point from each of the groups (i.e. control and stimulated). Data are reported as the mean \pm one standard deviation of the mean due to the large sample size $(n=30)$.

\section{Real time reverse transcriptase - polymerase chain reaction}

mRNA was extracted using trizol and collected using the Qiagen RNEasy Extraction kit (Qiagen, Valencia, CA). Samples were stored at $-80^{\circ} \mathrm{C}$ until assayed. cDNA was amplified with the TaqMan Universal PCR Master Mix (Applied Biosystems, Foster City, CA) and the ABI Prism 7000 Sequence Detection System (Applied Biosystems). RT-PCR determined mRNA expression of osteogenic differentiation markers, alkaline phosphatase (ALP Assay ID \#: Hs00240993_m1), and collagen type 1 (Col1 Assay ID \#: Hs00164004_a1), and stress response markers, heat shock protein 27 (hsp27 Assay ID \#: Hs00356629_g1) and heat shock protein 70 (hsp70 Assay ID \#: Hs00271244_s1). Assays on demand were purchased through Applied Biosystems (Carlsbad, CA). Relative gene expression was normalized to the housekeeping gene GAPDH (Assay ID \#: Hs99999905_m1) and calculated using the formula $2^{\text {(Ct value of GAPDH - Ct value of gene of interest) }}$ as previously used in our lab [28] and recommended by the manufacturer (Perkin Elmer User Bulletin \#2, Applied Biosystems, Foster City, CA). The threshold cycle $(\mathrm{Ct})$ was selected in the linear range of fluorescence for all genes.

\section{Metabolic activity}

AlamarBlue

For the purpose of assessing changes and differences in cellular activity, the control and stimulation groups were assayed every 5 days using AlamarBlue (Invitrogen Corp, Grand Island, NY). Briefly, a 9:1 (v/v) dilution of AlamarBlue to cell culture medium was added directly to the samples, then incubated for 2.5 hours. Following incubation, three $100 \mathrm{uL}$ replicates of media containing AlamarBlue was pipetted from each sample into a black 96 well plate. Fluorescence readings were taken using a plate reader at $560 \mathrm{~nm}$ excitation, $590 \mathrm{~nm}$ emission. Arbitrary units are determined as relative units of fluorescence intensity from the reduction of resazurin found in AlamarBlue to red 
fluorescent resorufin in the presence of metabolically active cells. Since AlamarBlue is nontoxic to cells, media that contained AlamarBlue was replaced with fresh differentiation medium following each reading. The same samples were measured at each time point. Excel was used to plot metabolic activity as a function of arbitrary units (AU) vs. time. Graphs were normalized to the fluorescence reading of the Alamar solution alone. Sample size per group per time point was $n=3$.

\section{Biochemical characterization}

Quantities of NADH, FAD, and lipofuscin found in osteogenically differentiating hMSCs were determined from analysis of endogenous TPEF images acquired on day 0 and every five days using a quantitative approach described in detail recently [29]. Briefly, images were taken at 860 and $755 \mathrm{~nm}$ excitation with emission filters centered at 525 and $455 \mathrm{~nm}$ as described in the morphology section. Images were filtered to remove noise and saturated pixels. The contribution from lipofuscin was determined as the fluorescence signal from the $455 \mathrm{~nm}$ channel at $860 \mathrm{~nm}$ excitation. For identifying the FAD contribution, a lipofuscin mask was created and multiplied with the fluorescence signal at $860 \mathrm{~nm}$ excitation, 525 emission; signal residing in pixels outside of the mask were assigned to FAD. NADH was calculated similarly by multiplying the lipofuscin mask with the $755 \mathrm{~nm}$ excitation, 455 emission channel, and considering the signal not emanating from pixels within the mask. The mask is the location of lipofuscin fluorescence as determined from data in the $455 \mathrm{~nm}$ channel at $860 \mathrm{~nm}$ excitation [29]. The mask was calculated as all pixels above a $10 \%$ threshold in the image [29]. This approach is reasonable because lipofuscin accumulates primarily in lysosomes, while NADH and FAD fluorescence originates predominantly from mitochondria [29]. Metabolic activity was determined through calculating the redox ratio as Redox Ratio $=$ FAD $/$ NADH. Decreases in the redox ratio yield increased metabolic activity, while a higher redox ratio reveals decreased metabolic activity. Unpaired t-tests were performed between the treated and untreated groups at each time point and between each time point per group. Sample size per group per time point was $n=3$.

\section{Protein deposition}

Images for observing type 1 collagen deposition were acquired through a non-destructive, non-linear optical scattering process, second harmonic generation (SHG) [30]. SHG images were acquired at $800 \mathrm{~nm}$ excitation in the forward scattering direction with the same system as the TPEF images using a $400 \mathrm{~nm} \pm 10 \mathrm{~nm}$ emission filter (Chroma, Rockingham, VT). Following imaging, collagen deposition was quantified through a fully automated adaptive threshold-based program written in MATLAB (MathWorks, Natick, MA), described in detail previously [31]. Sample size was $n=3$. The reported collagen fiber density refers to the percentage of pixels that are positive for SHG signal relative to the total number of pixels imaged within a field (i.e. a value of 0.1 corresponds to $10 \%$ of the pixels having SHG signal).

\section{Calcium staining}

To determine the presence of calcium, samples were washed with phosphate buffered saline, then fixed for 15 minutes using 1\% formaldehyde. Following fixation, samples from the control and stimulation groups were rinsed twice with water and soaked in an Alizarin Red solution for 10 minutes. Alizarin Red S (Sigma-Aldrich, St. Louis, MO) was 
weighed and diluted in deionized water to a final concentration of $0.8 \mathrm{~g}: 40 \mathrm{~mL}(\mathrm{w} / \mathrm{v})$. The solution $\mathrm{pH}$ was adjusted to 4.2. Samples were imaged in water using a Zeiss Axiovert S100 phase contrast microscope.

\section{Statistics}

Data are reported as the mean \pm one standard deviation unless otherwise noted. Data were analyzed using an unpaired two-tailed student $\mathrm{t}$-test, assuming equal variance with a $95 \%$ confidence interval. A p-value of $<0.05$ was considered statistically significant unless otherwise noted. In addition, for reporting a large number of comparisons between time points, the Bonferroni Correction factor was applied to correct for multiple comparisons of artifacts. For determining statistical significance using the Bonferroni Correction factor, alpha was set as $\alpha=0.05 / 10=0.005$.

\section{Results}

\section{Electric field effects on cell morphology}

On day 0 following initial stem cell seeding and attachment, TPEF images were taken for baseline morphological comparisons (Figure 2a). Cells in the control and stimulation groups appeared thin and elongated, a characteristic of undifferentiated hMSCs.

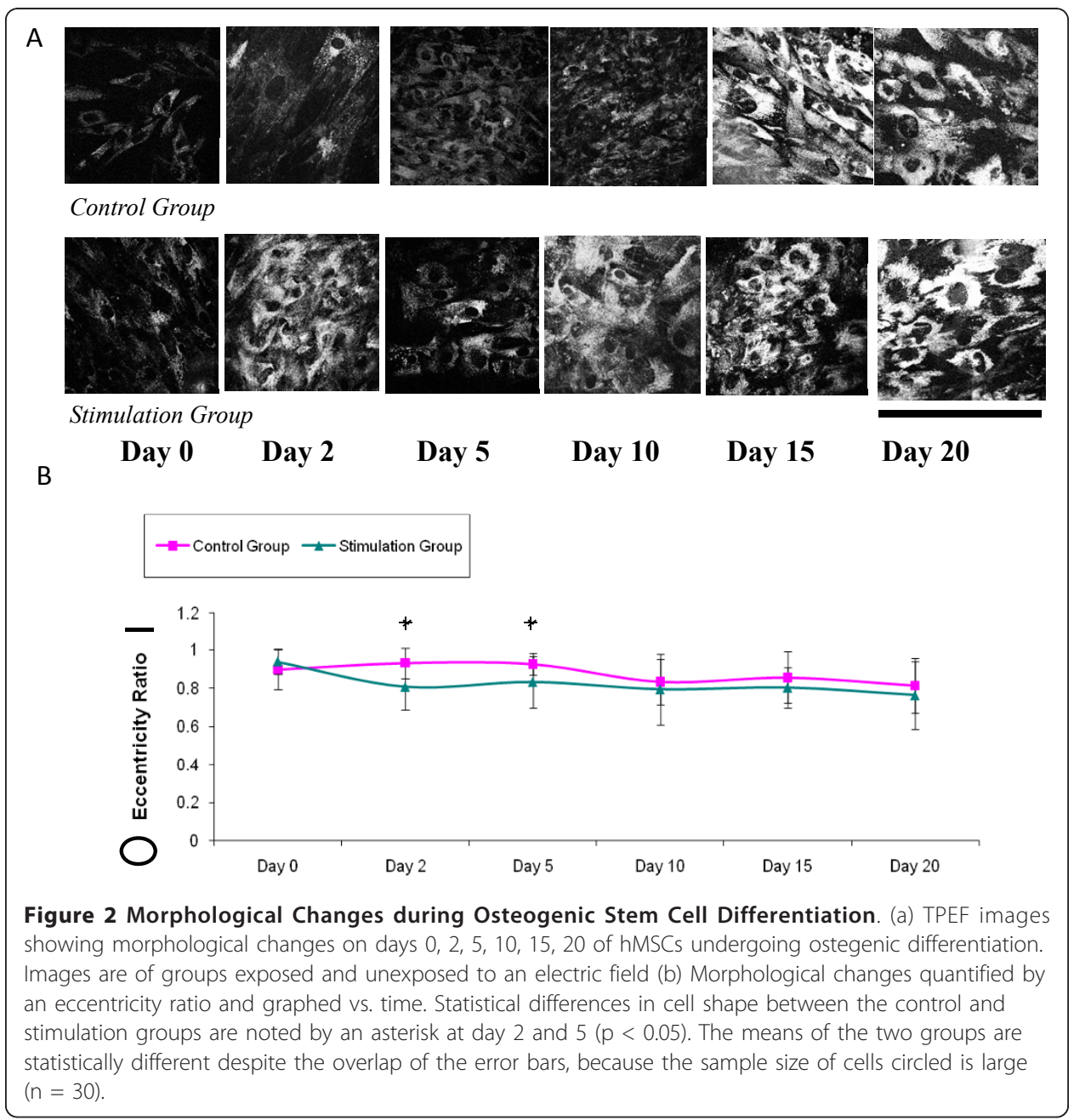


Throughout the experiment, both groups exhibited a distinct change in cellular phenotype. At the first two time points, stem cells exposed to an electric field appeared rounder than those in differentiation medium only and had an eccentricity value (Figure $2 \mathrm{~b}$ ) that was statistically lower than the control group $(\mathrm{p}<0.05)$. By day 10 , the cells in both groups exhibited a circular morphology. Quantitative morphological measures of eccentricity over 20 days are graphed in Figure $2 b$.

\section{Osteogenic markers}

Osteogenically differentiating hMSCs revealed overexpression of the early bone marker alkaline phosphatase (ALP) and mid marker type 1 collagen (col1). In the short term, on days 5 and 10, ALP and col 1 mRNA expression increased in the control group (Figure 3a, 3b) ( $\mathrm{p}<0.05$ ); osteogenically differentiating stem cells in the stimulation group showed enhanced ALP and col1 at later time points, days 15 and 20 (Figure 3a, 3b) with statistically significant differences in ALP expression at day 20 and col1 at day 15 and day 20. During the second week, ALP and col1 upregulation of the osteogenic differentiating stem cells in the stimulation group were two times greater than expression in the control group at day 10. At the end of week 2, the stimulation group had ALP and col1 upregulation statistically higher than the control $(\mathrm{p}<0.05)$.

\section{Stress response markers}

Heat shock protein 27 (hsp27), a stress response marker involved in osteogenic differentiation, was upregulated in the stimulation group on days 10, 15, and 20 when

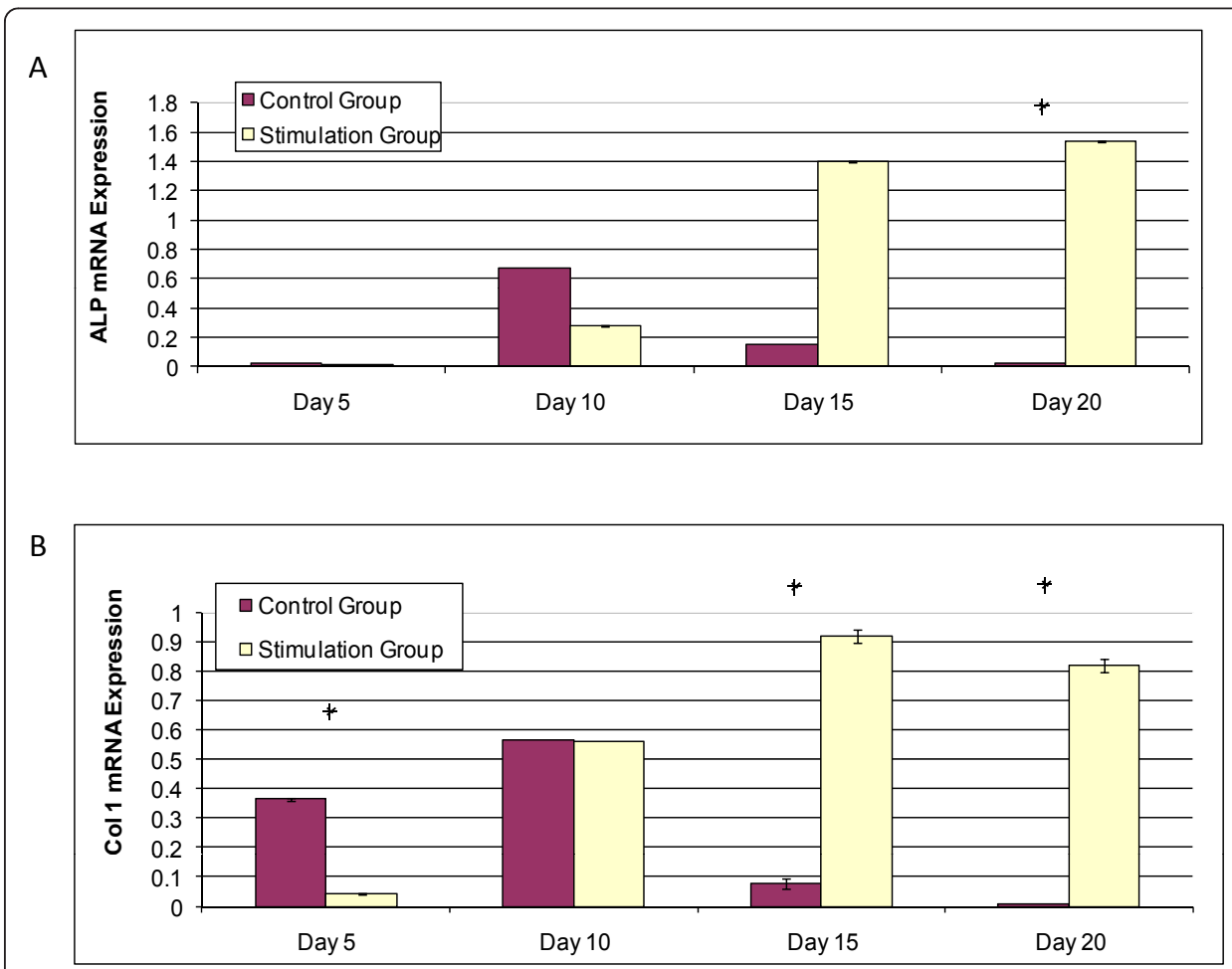

Figure 3 mRNA Expression Levels during Osteogenic Differentiation. (a) ALP mRNA expression relative to GAPDH vs. time. (b) Col1 mRNA expression relative to GAPDH vs. time. Statistical significance between groups is marked by an asterisk ( $p<0.05)$. 
compared to the control group (Figure 4a). Hsp27 upregulation was statistically significant compared to the control group on day 15 ( $\mathrm{p}<0.05)$. Hsp70, a protein involved in cellular metabolism, was upregulated in the stimulation group at day $20(\mathrm{p}<0.05)$, however consistent upregulation compared with controls was not observed (Figure 4b). In fact, Hsp70 expression was higher for the control groups on day 10 and 15.

\section{Cellular and Metabolic activity changes}

Increases in cellular activity were found using Alamar blue staining in both groups over the 20 day differentiation period (Figure 5$)$. Increased cellular activity $(\mathrm{p}<0.05)$ was identified in the control and stimulation groups from day 5 to day 10 and from day 10 to day 15 . There were not any significant changes in cellular activity identified between the two groups either on day 15 or day 20 .

Over the 20 day differentiation period, increased amounts of NADH and FAD were observed in the TPEF images in both the stimulation and control groups, consistent with the increased cellular activity results from the Alamar Blue studies (Figure 6). Increased contributions from lipofuscin were also observed throughout hMSC osteogenic differentiation, and were most prominent in the stimulation group (Figure 6). Increases in cell number were seen as well in TPEF images between days 0,5 , and 10 in both the control and stimulation groups. Significant enhancements in the levels of NADH and FAD were observed in the stimulation group compared to the control group during the first 10 days of treatment (Figure 7a-c). Indeed, the combination of $\mathrm{NADH}$ and FAD contributions to assess the redox ratio reveals changing metabolic activity for both the stimulation and control groups over the 20 days (Figure $7 \mathrm{~d}$ ). On day 15 and day 20, the redox ratio increases statistically significantly in the stimulation

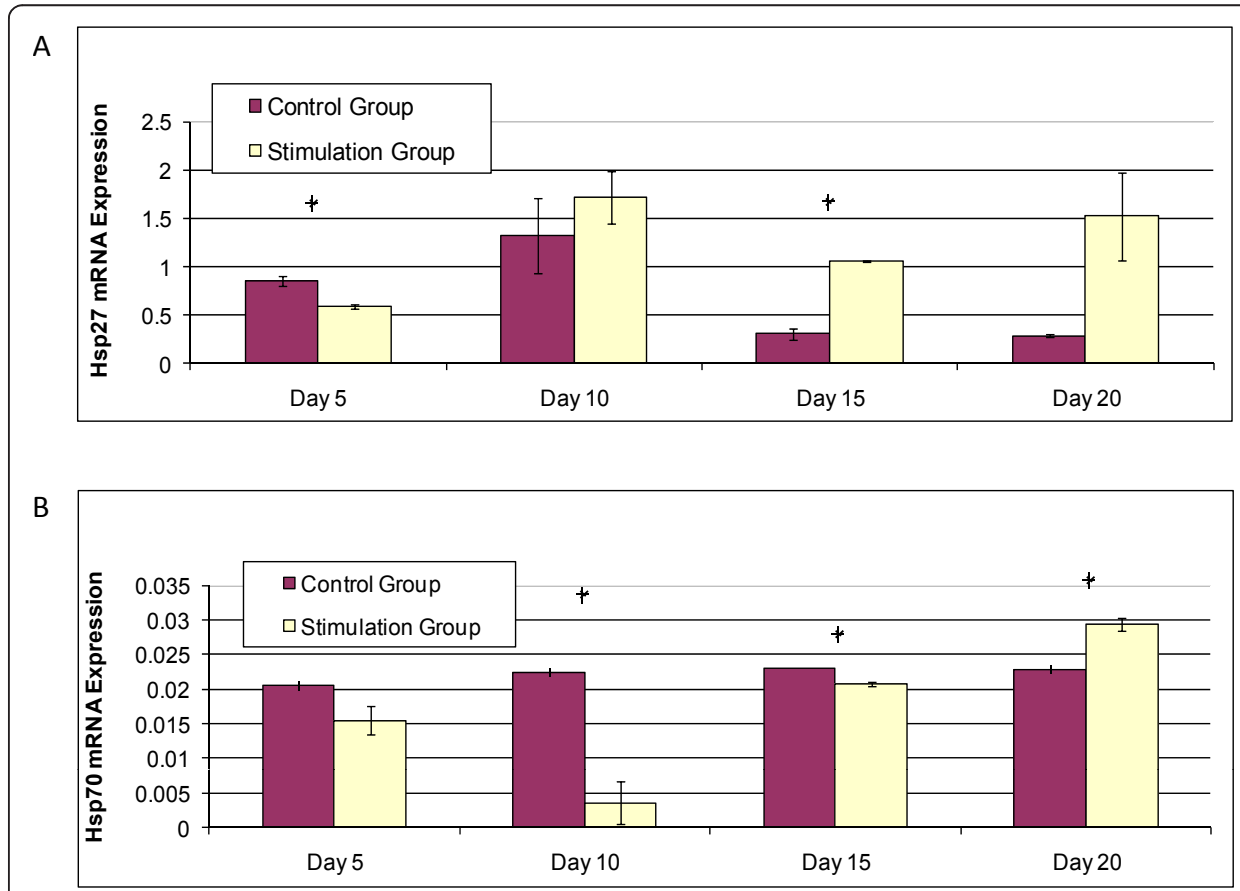

Figure 4 Heat Shock mRNA Expression. (a) Hsp27 mRNA expression relative to GAPDH vs. time. (b) Hsp70 mRNA expression relative to GAPDH vs. time. Statistical significance between groups is marked by an asterisk $(p<0.05)$. 

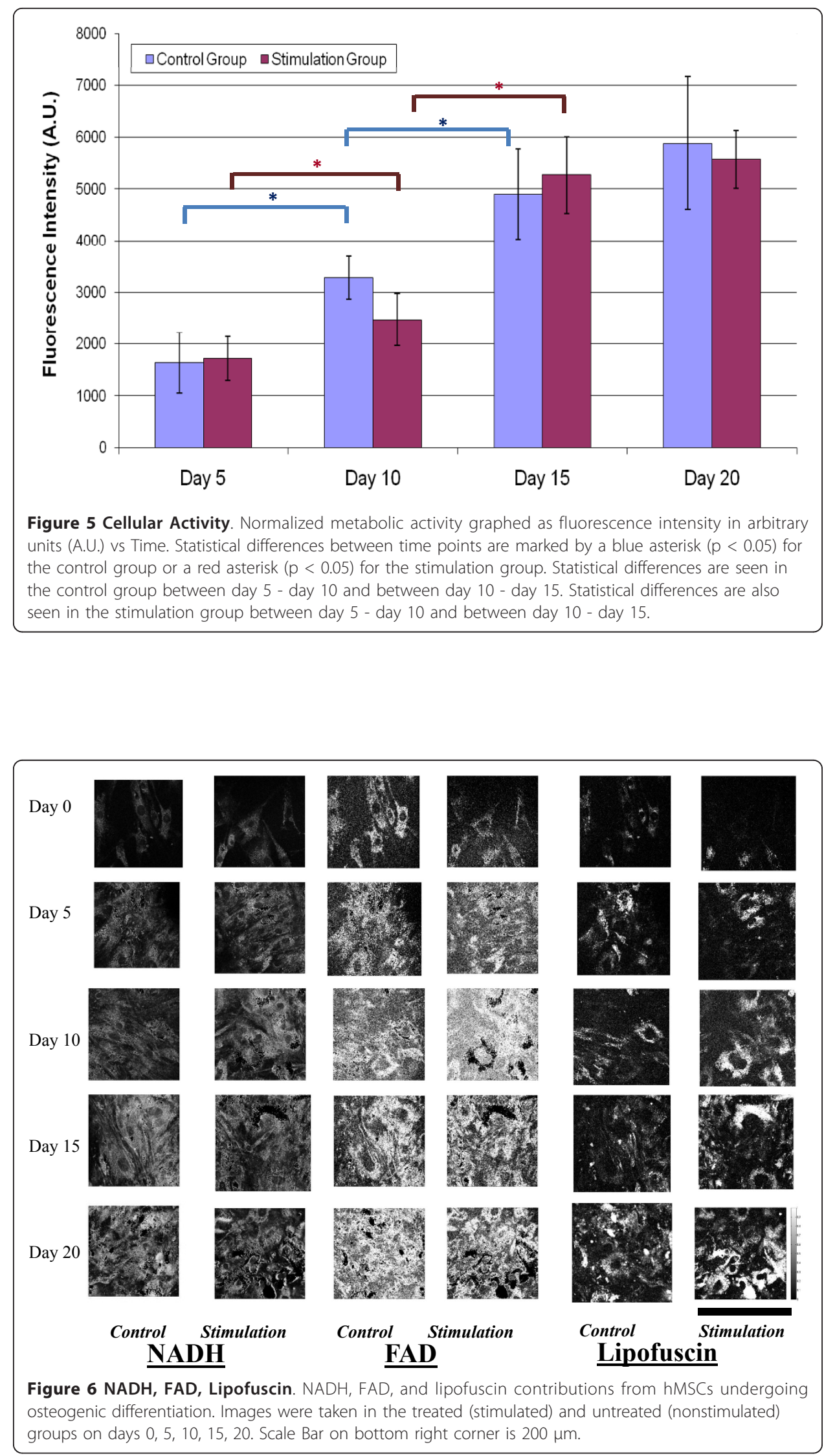


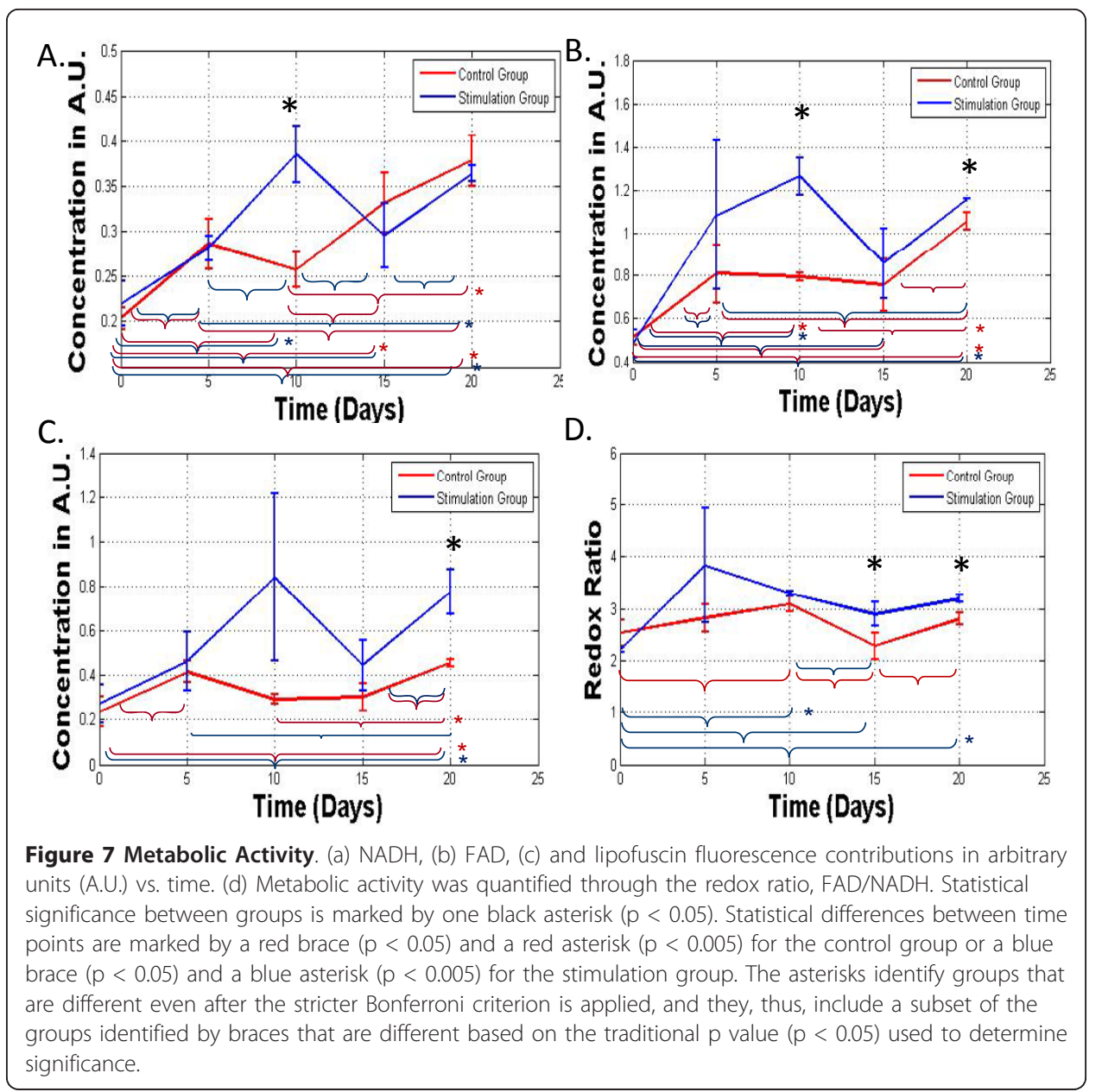

group compared to the controls $(\mathrm{p}<0.05)$ (Figure $7 \mathrm{~d}$ ). Interestingly, changes in lipofuscin observed in the stimulation group follow the trend of changes observed in hsp27 expression, suggesting a potential connection with oxidative stress mechanisms.

\section{Collagen deposition}

Type I collagen protein expression is one of several markers of hMSC-osteoblast differentiation. The lack of centro-symmetry in the collagen fibers results in significant levels of SHG. On day 0 and day 5, fibrillar protein deposition in the control group and stimulation groups was observed (Figure 8a). On day 15, protein deposition by SHG resembled collagen fibers in both the control group and stimulation groups with similar quantitative results (Figure $8 \mathrm{~b}$ ). The rate of change over 20 days in collagen deposition levels is more significant for the stimulation group $(\mathrm{p}<0.005)$, consistent with the observed upregulation of ALP and col I gene expression (Figure 8b).

\section{Bone mineralization}

On day 28, both the control and stimulation groups exhibited osteogenic differentiation potential towards bone, based on calcium deposition and calcium nodule formation (Figure 9). Calcium staining was compared to a stained negative control of non-differentiated hMSCs (image not shown). 


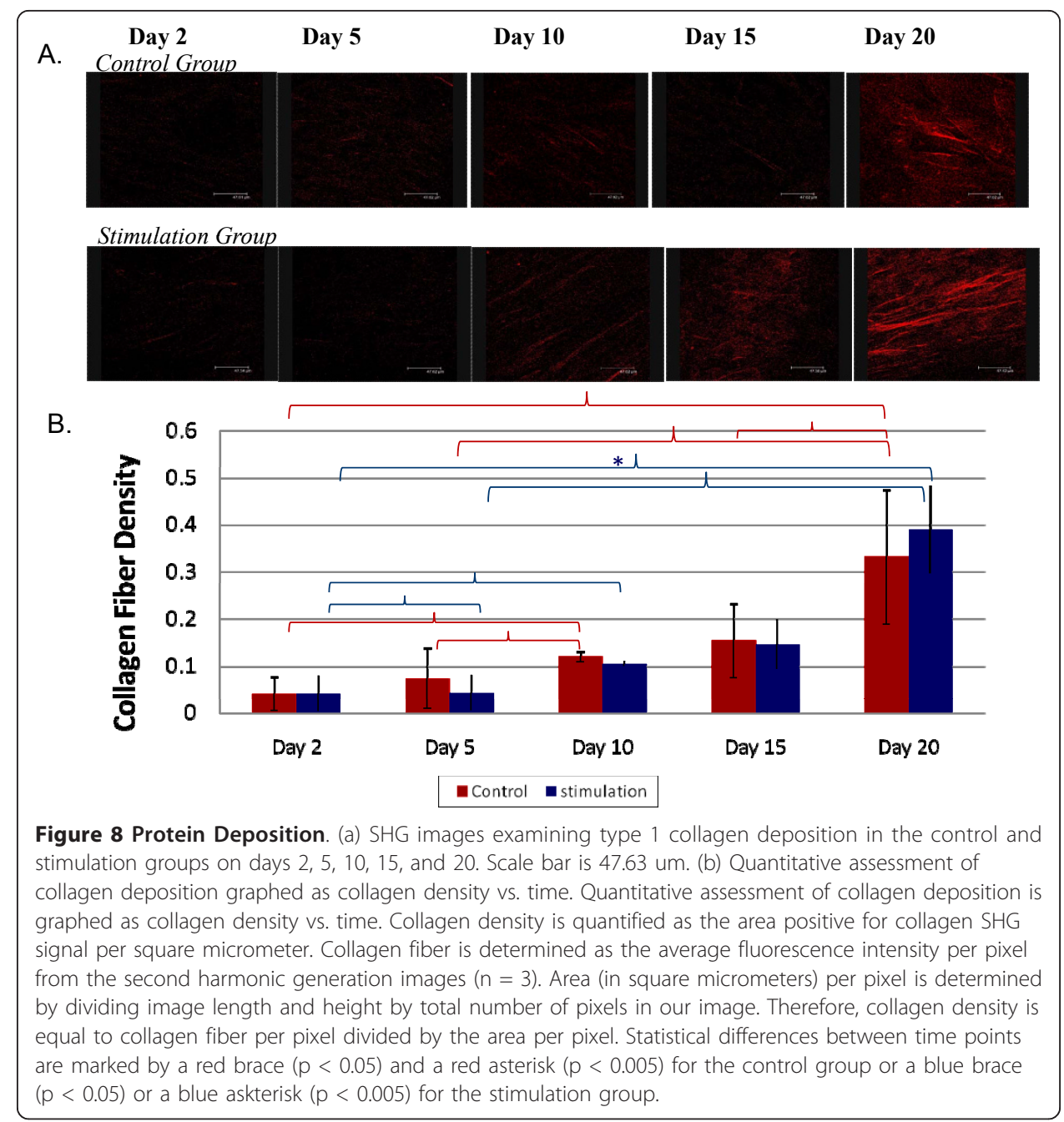

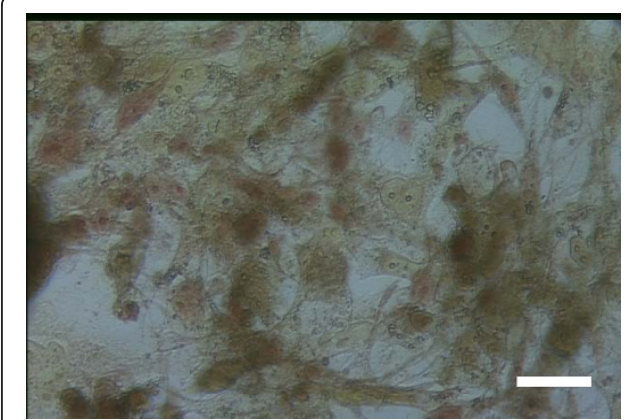

Control Group

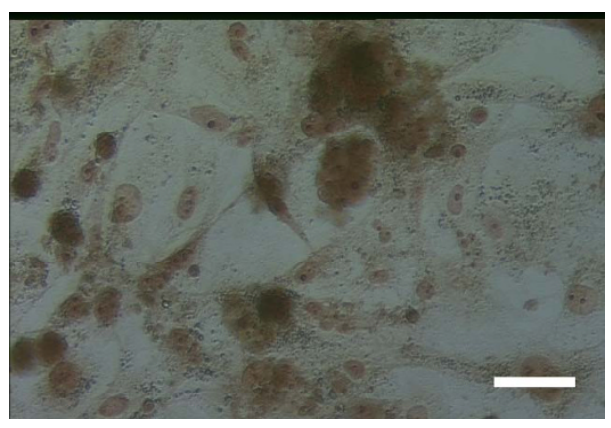

Stimulation Group

Day 28

Figure 9 Calcium Staining. Alizarin red stain at day 28 showing calcium deposition (red) in both the control group and the stimulation group. Scale bar is $50 \mathrm{um}$. 


\section{Discussion}

Many studies have revealed increased bone marker expression and increased proliferation on terminally differentiated osteoblasts following exposure to electric fields. For example, in one article, osteoblasts seeded in 3-dimensional constructs and exposed to a $75 \pm 2 \mathrm{~Hz}, 5 \pm 1 \mathrm{mV}, 2 \pm 0.2 \mathrm{mT}$ electric field expressed increased levels of decorin, osteocalcin, osteopontin, type I collagen, type III collagen, TGF-B, and fibronectin [32]. Levels of osteoblast expression markers increased 1.3 times, 12.2 times, 10.0 times and 10.5 times respectively [32]. Another study revealed increased proliferation up to $31 \%$ in osteoblasts exposed to a 2-day continuous $1.5 \mathrm{uA} / \mathrm{cm} 2 ; 3000 \mathrm{~Hz}$ electric field [33]. Recent studies have started to reveal responses of hMSCs to osteogenic differentiation in response to electric field stimulation [34-36]. For example, hMSCs isolated from bone marrow and exposed to a $7.5 \mathrm{~Hz}$ repetition rate of quasi-rectangular pulses revealed earlier increases in ALP gene expression as well as increases in of Runx2/ Cbfa1during the mid stage of differentiation [36]. In addition, calcium content was increased in the pulsed electromagnetic field treated cultures [36]. In another article, human mesenchymal stromal cells exposed to $100 \mathrm{~Hz}$ biphasic electric current at $1.5 \mathrm{uA} / \mathrm{cm} 2$ induced increased in vascular endothelial growth factor (VEGF) and (BMP)-2 expression [33]. However, in our present study utilizing ostegenic differentiating hMSCs additional assessments and insights are attained. Specifically, in our 28 day study, heat shock protein responses were correlated with osteogenic differentiation markers and field effects on cellular morphology were examined. Cellular activity was tracked as an indication of cell proliferation. Finally, novel optical methods were employed for monitoring specific osteogenic differentiation markers including fibrillar collagen deposition, morphological changes from an elongated hMSC phenotype to a circular osteogenic morphology, and changes in metabolic activity and lipofuscin.

Human mesenchymal stem cells are found in the stromal compartment of bone marrow and are highly proliferative. They are able to differentiate down several lineages including bone, cartilage, fat, and skeletal tissue and play important roles in fracture repair. Specifically, mesenchymal stem cells are a source of progenitors for osteoblast differentiation [37]. Differentiated osteoblasts are important during fracture repair as they contribute to a supply of intramembranous bone formation [37]. Since, previous results have shown that mesenchymal stem cells are the most competent to respond to biophysical input, including electric and electromagnetic fields [14], the application of electric fields on hMSCs may give us better quality of regrown bone and fracture repair.

\section{Morphological changes}

Electric fields are known to alter membrane morphology with regard to cell elongation, alignment, migration, adhesion, and other tissue responses including osteogenic differentiation [38-41]. During the first week of stimulation, namely on day 2 and day 5, eccentricity values describing cellular morphology were $13.2 \%$ and $10 \%$ lower, respectively, in the stimulation group compared to the control group (Figure 2a, 2b). One possible reason for the immediate morphological change is the extracellular force fields and stress placed on the plasma membrane $[42,43]$. 


\section{Osteogenic response}

The differentiation profile of the electrically stimulated group initially revealed a delayed response on days 5 and 10 in ALP and col1 mRNA expression compared to the non-stimulated group, keeping the cells in an undifferentiated state. At day 15, the stimulated group exhibited a $2 \times$ higher ALP and col1 expression level than the control group at peak levels. A later but more pronounced effect seen here with osteogenic markers may account for why electrical stimulation is effective and approved by the FDA for healing nonunions [36,44], even though it is not the first line of treatment following average fracture injuries.

\section{Heat shock response}

Heat shock protein expression is upregulated or changed when cells are under stress conditions, including oxidative damage and temperature elevation, for the purpose of maintaining cellular homeostasis [21]. Heat shock proteins participate in protein folding, degradation, and secretion [45]. Furthermore, heat shock proteins may also contribute to differentiation responses. For example, inducing mild heat stress of $41^{\circ} \mathrm{C}$ for 1 hour can modulate differentiation in normal human epidermal keratinocytes [22]. This effect is not unique to human cell differentiation. Small heat shock proteins, known to have molecular mass between 15 - $30 \mathrm{kDa}$, such as hsp27 are known to be expressed and regulated during differentiation and development in many organisms including humans, mice, and zebrafish [21]. More specifically, mild heat shock proteins can effect osteodifferentiation [21-23]. In the present study, heat shock protein 27 was upregulated starting on day 10 in the stimulation group (Figure 4a), while osteodifferentiation markers, ALP and col1, were upregulated soon after on day 15 and day 20 (Figure 3a, 3b). Not only did the electric field alter bone marker upregulation, yielding a different time stamp and magnitude on expression levels than in the controls, but the applied field also altered heat shock expression. While hsp27 expression preceded the osteodifferentiation response, recent literature reveals hsp70 involvement with metabolic activity [46-48].

Hsp70, like hsp27, is upregulated during heat and oxidative stress. For example, a sub-lethal heat stress of $42^{\circ} \mathrm{C}$ on rat hearts in vivo for 15 minutes induced hsp70 and vascular endothelial growth factor (VEGF) upregulation within 4 hours, promoting endothelial cell proliferation [46]. In another study, chondrocytes that had hsp70 mRNA transduced expressed higher metabolic activity under heat stress of $48^{\circ} \mathrm{C}$ [47]. Mild heat shock altered hsp70 expression in human bone marrow stromal cells and induced cell proliferation, ALP expression, and mineralization [49]. In the present studies, hsp70 expression was downregulated during the first 15 days of stimulation compared to the control group (Figure $4 \mathrm{~b}$ ). The redox ratio reveals metabolic activity was downregulated in the stimulation group on days 15 and days 20 ( $\mathrm{p}<0.05$ ) (Figure 7d). In the electrical stimulation group, hsp70 increased slightly, with a significant increase by day 20 (Figure 4b). Overall, cellular activity increased in both groups over 20 days based on the Alamar Blue assay (Figure 5). While results show upregulation in hsp27 on days 10,15 , and 20 , and upregulation in hsp70 by day 20 , the origins of these changes are not yet clear. While differentiation rate and response were affected, the possible relationships between applied electric field, stress response markers, and osteogenic markers, on osteodifferentiation will require further mechanistic insight. 


\section{NADH, FAD, Lipofuscin Contributions}

Noninvasive non-linear optical techniques and their combinations, such as second harmonic generation and two-photon excited fluorescence microscopy, provide information that is independent of cell source and environment on several areas that are of interest to tissue engineers, including but not limited to cellular and cell matrix interactions [50]. More specifically, non-linear optical techniques provide information on several aspects of cell and tissue fate including malignancy, cellular differentiation, proliferation, biochemical changes, and apoptosis [51,52]. We utilized a novel quantitative method developed recently [29] to determine the contributions of NADH, FAD and lipofuscin in cells treated with electrical field stimulation. Interestingly, enhancements in the levels of all three chromophores during the first 10 days of treatment, precede the observed upregulation in ALP and Col1 expression and are in agreement with early cellular morphological changes quantified by eccentricity values. The early changes we observe optically via entirely non-invasive means demonstrates the value of such methods as an effective characterization tool over expensive, time consuming biochemical assays and staining.

Over the 20 day differentiation period, we observe a small increase in the redox ratio for both groups, consistent with our previous osteogenic differentiation studies under normoxic conditions [24]. In addition, we find that the redox ratio is significantly higher for the stimulation than the control group. This may indicate that even though the cells are more active, they dedicate a higher percentage of their energy stores towards differentiation-related processes rather than oxidative phosphorylation.

\section{Cellular mechanisms}

It is known that BMP, wnt, and TGF- $\beta$ signaling pathways are involved in upregulating factors such as SMAD, Runx2, ALP, col1, osteocalcium, osteopontin, and kinases JNK and p38 during osteogenic differentiation [53-55]. During osteogenic differentiation, BMP-2,$3,-4,-5$ increase SMAD-1, $-3,-4,-5$, elevating the main transcription factor in osteogenic differentiation, Runx2 (Figure 10a). Runx2/cbfa1 upregulates the early osteogenic marker,

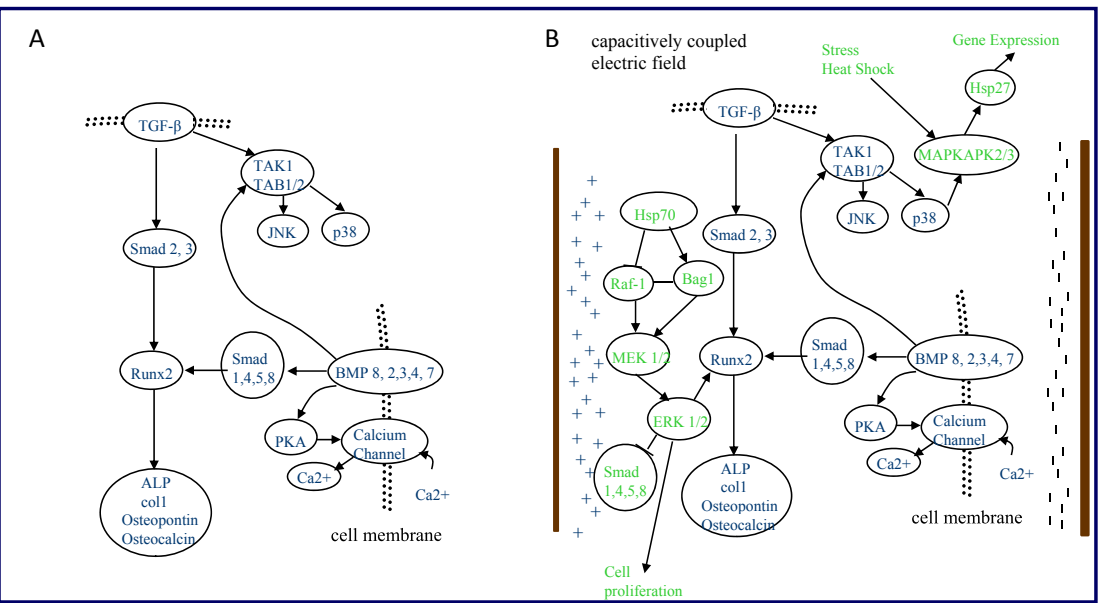

Figure 10 Cellular Mechanisms during Osteogenic Stem Cell Differentiation. (a) Osteogenic stem cell differentiation pathways (b) Osteogenic stem cell differentiation pathways and stress response roles in an alternating current electric field. 
ALP, mid marker col1, and late markers osteocalcin and osteopontin (Figure 10a) [53-55]. In addition, it has been demonstrated that osteoblast differentiation is mediated by opposing differentiation and proliferation signaling pathways, specifically ERK1/2 and PKB pathways [56]. The time for complete hMSC to osteoblast differentiation has been demonstrated over 4-5 weeks. In the present studies, $100 \mathrm{ng} / \mathrm{mL}$ BMP-2 was included to shorten the time to differentiation by acting directly on the BMP receptor.

Current hypotheses proposing how electric fields affect cell differentiation include altering membrane potential through hyperpolarization and depolarization $[57,58]$, modification of ion channels including density and distribution of receptors, calcium channel activation, and activating the extracellular-signal-regulated kinase (ERK) pathway $[38,59]$. We suggest two additional factors and their roles in electrical stimulation on osteodifferentiation: heat shock proteins 70 and 27 (Figure 10b).

Hsp70 has been reported to activate ERK1/2 pathways for controlling cell proliferation and survival. ERK1/2 activation can be achieved through Raf-1 and Bag1 activation [60]. Raf-1 and Bag1 upregulate MEK1/2 thus activating the ERK1/2 pathway [53-55,60-62]. The ERK pathway has a direct effect on cell proliferation [53-55,61,62] and was followed through our cellular and metabolic activity assays. Under extracellular stress conditions, heat shock proteins also upregulate mitogen activated protein kinase activated protein kinase 2/3 (MAPKAPK 2/3) upregulating hsp27 [63]. Hsp27 affects gene expression and differentiation, growth, and actin cytoskeleton reorganization $[64,65]$. In the present studies, hsp27 and hsp70 were upregulated in response to electric field application and compared with differentiation marker response, protein deposition, and cellular activity.

\section{Conclusions \& future directions}

Exposing hMSCs undergoing differentiation into osteoblasts in a $20 \mathrm{mV} / \mathrm{cm}$ oscillating $60 \mathrm{kHz}$ field for 40 minutes daily over 28 days increased hMSC osteogenic differentiation and activated osteogenic pathways. Future directions for this research include staining and quantifying for ALP and Hsp27 protein expression then correlating ALP and Hsp27 gene expression results to protein expression profiles. Additional transcription factors that are involved in osteogenic differentiation, such as Runx/Cbfa and bone sialoprotein (BSP), will be examined during electric field exposure.

\section{Appendix}

Electric field strength calculations from chamber center

Function for Gauss's Law

(for infinite line of charge)

$$
\overrightarrow{\mathrm{E}}=\frac{1}{2 \pi \varepsilon_{0}}\left(\frac{\rho}{r}\right)
$$

\section{Cartesion to Cylindrical Coordinates}

$$
\begin{aligned}
\mathrm{X} & =\mathrm{r} \cos \varphi \\
\mathrm{Y} & =\mathrm{r} \sin \varphi \\
\mathrm{Z} & =\mathrm{z}
\end{aligned}
$$




\section{Cylindrical to Cartesian Coordinates}

$$
\begin{aligned}
& r=\sqrt{\left(x^{2}+y^{2}\right)} \\
& \varphi=\tan ^{-1}\left(\frac{y}{x}\right)
\end{aligned}
$$

$$
\mathrm{Z}=\mathrm{z}
$$

\section{Convert function from Cylindrical to Cartesian Coordinates}

$$
\begin{aligned}
& \mathrm{E}_{\mathrm{x}}= \operatorname{Ar} \cos \varphi \\
& \mathrm{E}_{\mathrm{x}}= f(\mathrm{r}) \cos \varphi \\
& \mathrm{E}_{\mathrm{x}}=f\left(\sqrt{\left(x^{2}+y^{2}\right)}\right) \cos \varphi ; \\
& \mathrm{E}_{\mathrm{y}}= \mathrm{A}_{\mathrm{r}} \sin \varphi \\
& \mathrm{E}_{\mathrm{y}}= f(\mathrm{r}) \sin \varphi \\
& \mathrm{E}_{\mathrm{y}}=f\left(\sqrt{\left(x^{2}+y^{2}\right)}\right) \sin \varphi ; \\
& \mathrm{E}_{\mathrm{z}}= 0 ; \\
& \mathrm{E}_{\mathrm{x}}=f\left(\sqrt{\left(x^{2}+y^{2}\right)}\right) \cos \left(\tan ^{-1}(y / x)\right) ; \\
& \mathrm{E}_{\mathrm{y}}=f\left(\sqrt{\left(x^{2}+y^{2}\right)}\right) \sin \left(\tan ^{-1}(y / x)\right) ; \\
& \mathrm{E}_{\mathrm{z}}=0 ;
\end{aligned}
$$

Trigonometric Identities

$$
\begin{aligned}
& r=\sqrt{\left(x^{2}+y^{2}\right)} \\
& \cos \left(\tan ^{-1} \mathrm{x}\right)=\frac{1}{\sqrt{x^{2}+1}} \\
& \sin \left(\tan ^{-1} \mathrm{x}\right)=\frac{x}{\sqrt{x^{2}+1}}
\end{aligned}
$$

Replace $\mathrm{r}$ with $\sqrt{\left(x^{2}+y^{2}\right)}$

$$
\begin{aligned}
& \mathrm{E}_{\mathrm{x}}=\frac{1}{2 \pi \varepsilon_{0}}\left(\frac{\rho}{\sqrt{x^{2}+y^{2}}}\right)\left(\frac{1}{\sqrt{\left(\frac{y}{x}\right)^{2}+1}}\right)\left(\frac{x}{x}\right) \\
& \mathrm{E}_{\mathrm{x}}=\frac{\rho}{2 \pi \varepsilon_{0}}\left(\frac{x}{x^{2}+y^{2}}\right)
\end{aligned}
$$




$$
\begin{aligned}
& \mathrm{E}_{\mathrm{y}}=\frac{1}{2 \pi \varepsilon_{0}}\left(\frac{\rho}{\sqrt{x^{2}+y^{2}}}\right)\left(\frac{y}{x}\right)\left(\frac{1}{\sqrt{\left(\frac{y}{x}\right)^{2}+1}}\right)\left(\frac{x}{x}\right) \\
& \mathrm{E}_{\mathrm{y}}=\frac{\rho}{2 \pi \varepsilon_{0}}\left(\frac{y}{x^{2}+y^{2}}\right)
\end{aligned}
$$

$\mathrm{E}_{\mathrm{z}}=0$;

Offset two infinite line charges by distance $\left[+\frac{d}{2},-\frac{d}{2}\right]$

$$
\begin{aligned}
& \mathrm{E}_{\mathrm{x}}=\frac{\rho}{2 \pi \varepsilon_{0}}\left(\frac{x-d / 2}{(x-d / 2)^{2}+y^{2}}\right)+\frac{\rho}{2 \pi \varepsilon_{0}}\left(\frac{x+d / 2}{(x+d / 2)^{2}+y^{2}}\right) \\
& \mathrm{E}_{\mathrm{y}}=\underbrace{\frac{\rho}{2 \pi \varepsilon_{0}}\left(\frac{y}{(x-d / 2)^{2}+y^{2}}\right)+\underbrace{\frac{\rho}{2 \pi \varepsilon_{0}}}\left(\frac{y}{(x+d / 2)^{2}+y^{2}}\right)}
\end{aligned}
$$

Find equation for voltage Drop Between Rods

$$
\mathrm{V}_{\mathrm{ab}}=\int E_{x} \bullet d \ell
$$

E field component in the $\mathrm{x}$-direction between two carbon rods ( $E$ field in $y$-direction is zero)

$$
\begin{aligned}
& \mathrm{V}_{\mathrm{ab}}=\int_{\left(\frac{-d}{2}\right)+a}^{\left.\frac{d}{2}\right)^{-a}} \frac{\rho}{2 \pi \varepsilon_{0}}\left[\frac{1}{\left(x-\frac{d}{2}\right)}-\frac{1}{\left(x+\frac{d}{2}\right)}\right] \mathrm{dx} \\
& \mathrm{V}_{\mathrm{ab}}=\left.\frac{\rho}{2 \pi \varepsilon_{0}}\left(\ln \left[x-\frac{d}{2}\right]-\ln \left[x+\frac{d}{2}\right]\right)\right|_{\left(\frac{d}{2}+a\right)} ^{\left(\frac{d}{2}+a\right)} \\
& \mathrm{V}_{\mathrm{ab}}=\frac{\rho}{2 \pi \varepsilon_{0}}\left(\ln \frac{\left[x-\frac{d}{2}\right]}{\left[x+\frac{d}{2}\right]}\right)\left(\begin{array}{l}
\left(\frac{d}{2}-a\right) \\
\left(\frac{-d}{2}+a\right)
\end{array}\right.
\end{aligned}
$$




$$
\begin{aligned}
& \mathrm{V}_{\mathrm{ab}}=\frac{\rho}{2 \pi \varepsilon_{0}}\left\{\left\{\left(\frac{\ln [-a]}{[d-a]}\right)-\left(\frac{\ln [-d+a]}{[a]}\right)\right\}\right. \\
& \mathrm{V}_{\mathrm{ab}}=\frac{\rho}{2 \pi \varepsilon_{0}} \ln \frac{[-a][a]}{[d-a][a-d]} \\
& \mathrm{V}_{\mathrm{ab}}=\ln \frac{-\left[a^{\wedge} 2\right]}{[d-a][a-d]} \\
& \mathrm{V}_{\mathrm{ab}}=\frac{\rho}{2 \pi \varepsilon_{0}} \ln \frac{a^{\wedge} 2}{\left[(d-a)^{\wedge} 2\right]} \\
& \mathrm{V}_{\mathrm{ab}}=\ln (x)^{\wedge} 2=2 \ln (x) \\
& \mathrm{V}_{\mathrm{ab}}=\frac{\rho}{2 \pi \varepsilon_{0}} 2 * \ln \frac{a}{[(d-a)]} \\
& \rho=\frac{\mathrm{V}_{\mathrm{ab}} \pi \varepsilon_{0}}{\ln \left(\frac{a}{d-a}\right)}
\end{aligned}
$$

Substitute for charge density, $\rho$

$$
\begin{aligned}
& E_{x}=\frac{V_{a b}}{2 \ln \left(\frac{a}{d-a}\right)}\left[\frac{x-\frac{d}{2}}{\left(x-\frac{d}{2}\right)^{2}+y^{2}}-\frac{x+\frac{d}{2}}{\left(x+\frac{d}{2}\right)^{2}+y^{2}}\right] \\
& E_{\gamma}=\frac{V_{a b} y}{2 \ln \left(\frac{a}{d-a}\right)}\left[\frac{1}{\left(x-\frac{d}{2}\right)^{2}+y^{2}}-\frac{1}{\left(x+\frac{d}{2}\right)^{2}+y^{2}}\right]
\end{aligned}
$$

\section{Acknowledgements}

The authors would like to thank Lisa R Yanushefski for assistance with data collection. This research was supported by the Tissue Engineering Resource Center (TERC) through the NIH grant P41EB002520 from the National Institute of Biomedical Imaging and Bioengineering, NSF Grant No. BES0547292, NIH Grant No. R01EB007542, and a Tufts University Graduate Research Award.

\section{Authors' contributions}

MHT carried out the experiments, completed data analysis, and drafted the manuscript. WLR developed the algorithms for optical image analysis, assisted in imaging training and was involved in key intellectual discussions. MCG supervised electric field calculations. IG and DLK conceived of and supervised the project. IG and DLK supervised manuscript writing and revisions. All authors read and approved the final manuscript.

\section{Competing interests}

The authors declare that they have no competing interests. 


\section{References}

1. Praemer A, Furner S, Rice D: Musculoskeletal Conditions in the United States. Park Ridge, Il; 1992.

2. Kawamura K, Chung K: Treatment of scaphoid fractures and nonunions. J Hand Surg Am 2008, 33:988-997.

3. Novicoff W, Manaswi A, Hogan M, Brubaker S, Mihalko W, Saleh K: Critical analysis of the evidence for current technologies in bone-healing and repair. J Bone Joint Surg Am 2008, 90:85-81.

4. Hadjiargyrou M, Mcleod K, Ryaby J, Rubin C: Enhancement of fracture healing by low intenisty ultrasound. Clin Orthop 1998, 355s:s216-s229.

5. Azuma $Y$, Ito $M$, Harada $Y$, Takagi $H$, Ohta $T$, Jingushi S: Low-intensity pulsed ultrasounds accelerates rat femoral fracture healing by acting on the various cellular reactions in the fracture callus. J Bone Miner Res 2001, 16:671-680.

6. Ciombor D, Aaron R: The role of electrical stimulation in bone repair. Foot Ankle Clin 2005, 10:579-593.

7. Gan J, Glazer P: Electrical stimulation therapies for spinal fusions: current concepts. Eur Spine J 2006, 15:1301-1311.

8. Physio-Stim. [http://www.orthofix.com/products/physio-stim_hip.asp].

9. SpinalPakll Spine Fusion Stimulator. [http://www.biomet.com/spine/products.cfm?pdid=3\&majcid=11\&prodid=12].

10. Osteogen Bone Growth Stimulator. [http://www.biomet.com/trauma/products.cfm?pdid=4\&majcid=47\&prodid=268].

11. Osteogen Dual Lead Bone Growth Stimulator. [http://www.biomet.com/trauma/products.cfm?pdid=4\&majcid=47\& prodid=269].

12. EBI Bone Healing System. [http://www.biomet.com/trauma/products.cfm?pdid=4\&majcid=47\&prodid=267].

13. Britghton C, Pollack S: Treatment of Recalcitrant Non-Union with a Capactively Coupled Electrical Field. A Preliminary Report. The Journal of Bone \& Joint Surgery 1985, 67:577-585.

14. Aaron R, Ciombor D, Wang S, Simon B: Clinical biophysics:The promotion of skeletal repair by physical forces. Annals New York Academy of Sciences 2006, 1068:513-531.

15. Aaron R, Ciombor D: Acceleration of experimental endochondral ossification by biophysical stimulation of the progenitor cell pool. Journal of Orthopaedic Research 1996, 14:582-589.

16. Xu J, Wang W, Clark C, Brighton C: Signal Transduction in Electrically Stimulated Articular Chondrocytes Involves Translocation of Extracellular Calcium Through Voltage-Gated Channels. Osteoarthritis Cartilage 2009, 17:397-405.

17. Wang W, Wang Z, Zhang G, Clark C, Brighton C: Up-regulation of chondrocyte matrix genes and products by electric fields. Clinical Orthopaedics and Related Research 2004, 427:s163-s173.

18. Zhuang H, Wang W, Seldes R, Tahernia D, Fan H, Brighton C: Electrical stimulation induces level of TGF-B1 mRNA in Osteoblastic cells by a mechanism involving calcium/calmodulin pathway. Biochem Biophys Res Commun 1997, 237:225-229

19. Lorich D, Brighton C, Gupta R, Corsetti J, Levine S, Gelb I, Seldes R, Pollack S: Biochemical pathway mediating the response of bone cells to capacitive coupling. Clinical Orthopaedics and Related Research 1998, 350:246-256.

20. Brighton C, Wang W, Clark C: The Effect of Electrical Fields on Gene and Protein Expression in Human Osteoarthritic Cartilage Explants. J Bone Joint Surg Am 2008, 90:833-848.

21. Brown D, Christine K, Showell C, Conlon F: Small heat shock protein Hsp27 is Required for Proper Heart Tube Formation. Genesis 2007, 45:667-678.

22. Berge $U$, Kristensen $P$, Rattan S: Hormetic modulation of differentiation of normal human epidermal keratinocytes undergoing replicative senescence in vitro. Experimental Gerontology 2008, 43:658-662.

23. Norgaard R, M K, Rattan S: Heat Shock-Induced Enhancement of Osteoblastic Differentiation of hTERT-Immortalized Mesenchymal Stem Cells. Ann N Y Acad Sci 2006, 1067:443-447.

24. Tandon N, Cannizzaro C, Choh, Maidhof R, Marsano A, Au H, Radisic M, Vunjak-Novakovic G: Electrical stimulation systems for cardiac tissue engineering. Nat Protoc 2009, 4:155-173.

25. Sundelacruz S, Levin M, Kaplan D: Membrane Potential Controls Adipogenic and Osteogenic Differentiation of Mesenchymal Stem Cells. PLoS One 2008, 3:e3737.

26. Karageorgiou V, Meinel L, Hofmann S, Malhotra A, Volloch V: Bone Morphogenetic Protein-2 Decorated Silk Fibroin Films Induce Osteogenic Differentiation of Human Bone Marrow Stromal Cells. J Biomed Mater Res A 2004, 71:528-537.

27. Karageorgiou V, Tomkins M, Fajardo R, Meinel L, Snyder B, Wade K, Chen J, Vunjak-Novakovic G, Kaplan D: Porous Silk Fibroin 3-D Scaffolds for Delivery of Bone Morphogenetic Protein-2 In Vitro and In Vivo. J Biomed Mater Res A 2006, 78:324-334.

28. Mauney J, Nguyen T, Gillen K, Kirker-Head C, Gimble J, Kaplan D: Engineering adipose-like tissue in vitro and in vivo utilizing human bone marrow and adipose-derived mesenchymal stem cells with silk fibroin 3D scaffolds. Biomaterials 2007, 28:5280-5290.

29. Rice W, Kaplan D, Georgakoudi I: Two-Photon Microscopy for Non-Invasive, Quantitative Monitoring of Stem Cell Differentiation. PLoS One 2010, 5:e10075.

30. Rice W, Firdous S, Gupta S, Hunter M, Foo C, Wang Y, Kim H, Kaplan D, Georgakoudi I: Non-invasive characterization of structure and morphology of silk fibroin biomaterials using non-linear microscopy. Biomaterials 2008, 29:2015-2024.

31. Bayan C, Levitt J, Miller E, Kaplan D, Georgakoudi I: Fully automated, quantitative, noninvasive assessment of collagen fiber content and organization in thick collagen gels. Journal of Applied Physics 2009, 105:102042.

32. Fassina L, Visai L, Benazzo F, Bennedetti L, Calligaro A, De Angelis M, Farina A, Maliardi V, Margenes G: Effects of electromagnetic stimulation on calcified matrix production by SAOS-2 cells over a polyurethane porous scaffold. Tissue Eng 2006, 12:1985-1999.

33. Kim I, Song J, Zhang Y, Lee $Y$, Cho T, Song Y, Kim dK, Kim S, Hwang S: Biphasic Electric Current Stimulates Proliferation and Induces VEGF Productino in Osteoblasts. Biochim Biophys Acta 2006, 1763:907-916.

34. Kim I, Song J, Song Y, Cho T, Lee T, Lim S, Kim S, Hwang S: Novel effect of biphasic electric current on in vitro osteogenesis and cytokine production in human mesenchymal stem cells. Tissue Engineering Part A 2009, 15:2411-2422. 
35. Sun S, Liu Y, Lipsky S, Cho M: Physical Manipulation of calcium oscillations facilitates osteodifferentiation of human mesenchymal stem cells. FASEB J 2007, 21:1472-1480.

36. Tsai M, Li W, Tuan R, Chang W: Modulation of osteogenesis in human mesenchymal stem cells by a pulsed electromagnetic field stimulation. Journal of Orthopaedic Research 2009, 27:1169-1174.

37. Bielby R, Jones E, McGonagle D: The Role of Mesenchymal Stem Cells in Maintenance and Repair of Bone. Injury, Int J Care Injured 2007, 38S1:S26-S32.

38. Jahns M, Lou E, Durdle N, Bagnall K, Raso J, Cinats D, Barley R, Cinats J, Jomha N: The effect of pulsed electromagnetic fields on chondrocyte morphology. Medical and Biological Engineering \& Computing 2007, 45:917-925.

39. Rice W, Kaplan D, Georgakoudi I: Quantitative biomarkers of stem cell differentiation based on intrinsic two-photon excited fluorescence. J Biomed Opt 2007, 12:060504.

40. Dube J, Methot S, Moulin V, Goulet D, Bourdage M, Auger F, Germain L: External Electric fields induce morphological changes on human skin cells cultured in vitro. USRI 2005.

41. Sun S, Titushkin I, Cho M: Regulation of Mesenchymal Stem Cell Adhesion and Orientation in 3D Collagen Scaffold by Electrical Stimulus. Bioelectrochemistry 2006, 69:133-141.

42. Panagopoulos D, Karabarbounis A, Margaritis L: Mechanism for action of electromagnetic fields on cells. Biochem Biophys Res Commun 2002, 298:95-102.

43. Panagopoulos D, Messini N, Karabarbounis A, Philippetis A, Margaritis L: A mechanism for action of oscillating electric fields on cells. Biochem Biophys Res Commun 2000, 272:634-640.

44. Tsai M, Chang W, Chang K, Hou R, Wu T: Pulsed electromagnetic fields affect osteoblast proliferation and differentiation in bone tissue engineering. Bioelectromagnetics 2007, 28:519-528.

45. Jakob U, Gaestel M, Engel K, Buchner J: Small heat shock proteins are molecular chaperones. J Biol Chem 1993, 268:1517-1520

46. Gong B, Asimakis G, Chen Z, Albrecht T, Boor P, Pappas T, Bell B, Motamedi M: Whole-body hyperthermia induces upregulation of vascular endothelial growth factor accompanied by neovascularization in cardiac tissue. Life Sciences 2006, 79:1781-1788.

47. Kubo T, Arai Y, Takahashi K, Ikeda T, Ohashi S, Kitajima I, Mazda O, Takigawa M, Imanishi J, Hirasawa Y: Expression of transduced HSP70 gene protects chondrocytes from stress. J Rheumatol 2001, 28:330-335.

48. Laubitz D, Jankowska A, Sikora A, Wolinski J, Zabielski R, Grzesiuk E: Gut Myoelectrical activity induces heat shock response in Escherichia coli and Caco-2 Cells. Experimental Physiology 2006, 91:867-875.

49. Shui C, Scutt A: Mild heat shock induces proliferation, alkaline phosphatase activity, and mineralization in human bone marrow stromal cells and Mg-63 cells in vitro. J Bone Miner Res 2001, 16:731-741.

50. Georgakoudi I, Rice W, Hronik-Tupaj M, Kaplan D: Optical Spectroscopy and Imaging for the Non-Invasive Evaluation of Engineered Tissues. Tissue Engineering Part A 2008, 14:321-340.

51. Rice W, Kaplan D, Georgakoudi I: Quantitative biomarkers of stem cell differentiation based on intrinsic two-photon excited fluorescence. Book Quantitative biomarkers of stem cell differentiation based on intrinsic two-photon excited fluorescence 2007, 12:060504, City.

52. Levitt J, Hunter M, Mujat C, McLaughlin-Drubin M, Munger K, Georgakoudi I: Diagnostic cellular organization features extraced from autofluorescence images. Optics Letters 2007, 32:3305-3307.

53. Walker D, Wright N: Bone Morphogenetic Proteins and Spinal Fusion. Neurosurg Focus 2002, 13:1-13,

54. Qi H, Aguiar D, Williams S, La Pean A, Pan W, Verfaillie C: Identification of genes responsible for osteoblast differentiation from human mesodermal progenitor cells. PNAS 2003, 100:3305-3310.

55. BMP Pathway. [http://www.qiagen.com/geneglobe/pathwayview.aspx?pathwaylD=65].

56. Raucci A, Bellosta P, Grassi R, Basilico C, Manuskhani A: Osteoblast Proliferation or Differentiation is Regulated by Relative Strengths of Opposing Signaling Pathways. J Cel Physiol 2008, 215:442-451.

57. Sauer H, Rahimi G, Hescheler J, Wartenberg M: Effects of electrical fields on cardiomyocyte differentiation of embryonic stem cells. journal of cellular biochemistry 1999, 75:710-723.

58. Gross $D$, Loew $L$, Webb W: optical imaging of cell membrane potential changes induced by applied electric fields. Biophysical Journal 1986, 50:339-248.

59. Aaron R, Boyan B, Ciombor D, Schwartz Z, Simon B: Stimulation of Growth Factor Synthesis by Electric and Electromagnetic Fields. Clin Orthop 2004, 419:30-37.

60. Wang H, Takayama S, Rapp U, Reed J: Bcl-2 interactinv protein, BAG-1, binds to and activates the kinase Raf-1. Proc Natl Acd Sci 1996, 93:7063-7068

61. Reilly G, Golden E, Grasso-Knight G, Leboy P: Differential effects of ERK and p38 signaling in BMP-2 stimulated hypertrophy of cultured chick sternal chondrocytes. Cell Communication and Signaling 2005, 3:3.

62. Mossner D, Morimoto R: Molecular Chaperones and the stress of oncogenesis. Oncogene 2004, 23:2907-2918.

63. Maizels E, Peters C, Kline M, Cutler R, Shanmugan M, Hunzicker-Dunn M: Heat-Shock protein-25/27 phosphorylation by the isoform of protein kinase C. Biochem J 1998, 332:703-712.

64. Matsumoto T, Claesson-Welsh L: VEGF Receptor Signal Transduction. Sci STKE 2001, 112:re21.

65. Denhardt D: Signal-transducing protein phosphorylation cascades mediated by Ras/Rho proteins in the mammalian cell: the potential for multiplex signaling. Biochem J 1996, 318:729-747.

doi:10.1186/1475-925X-10-9

Cite this article as: Hronik-Tupaj et al:: Osteoblastic differentiation and stress response of human mesenchymal stem cells exposed to alternating current electric fields. BioMedical Engineering OnLine 2011 10:9. 